\title{
NANOELECTROMECHANICAL SILICON CARBIDE RESONATORS FOR ULTRA HIGH FREQUENCY APPLICATIONS
}

\author{
X.M.H. Huang, K.L. Ekinci, and Y.T. Yang \\ Condensed Matter Physics, California Institute of Technology \\ Pasadena, California 91125
}

C.A. Zorman and M. Mehregany

Electrical Engineering and Computer Science

Case Western Reserve U., Cleveland, OH 44106

\section{ABSTRACT}

Nanomechanical resonators with fundamental mode resonance frequencies in the Ultra High Frequency (UHF) band were fabricated from single crystalline $3 \mathrm{C}-\mathrm{SiC}$ thin films epitaxially grown on $\mathrm{Si}$ substrates using a combination of electron beam lithography and micromachining techniques. Resonant frequencies of doubly clamped resonator pairs were measured by magnetomotive transduction combined with a balanced bridge readout circuit. Resonant frequencies as high as $632 \mathrm{MHz}$ were recorded.

\section{INTRODUCTION}

The rapidly growing interest in RF MEMS has created a need to push the frequencies of micromechanical resonators into the UHF band. Evidence is emerging that polysilicon at best provides limited performance at these frequencies. As a result, recent initiatives are exploring structures made from materials with higher acoustic velocities [1-2], as well as nanometer-scale dimensions [3] to create devices with such frequencies. This paper reports on our latest efforts in developing nanometer-scale, electromechanical resonators using 3C-SiC thin films [4], employing a strategy that capitalizes on both a reduction in scale and an increase in acoustic velocity to achieve resonant frequencies in the UHF band.

\section{EXPERIMENTAL DETAILS}

The 3C-SiC films used in this work are heteroepitaxially grown on $100 \mathrm{~mm}$-diameter (100) $\mathrm{Si}$ wafers in a rf-inductionheated, APCVD reactor [5]. $\mathrm{SiH}_{4}$ and $\mathrm{C}_{3} \mathrm{H}_{8}$ are used as precursors, and $\mathrm{H}_{2}$ is used as a carrier gas. The epitaxial process is a two-step, high-temperature $(1280 \diamond \mathrm{C})$ procedure, involving the carbonization of the $\mathrm{Si}$ surface in $\mathrm{C}_{3} \mathrm{H}_{8} / \mathrm{H}_{2}$ ambient followed by epitaxial growth using $\mathrm{SiH}_{4}, \mathrm{C}_{3} \mathrm{H}_{8}$, and $\mathrm{H}_{2}$. The epitaxial growth recipe is optimized for micron-thick films yet produces $50 \mathrm{~nm}$ to $250 \mathrm{~nm}$-thick films of sufficient surface quality for e-beam lithography.

Device fabrication utilizes a combination of optical and electron beam lithography techniques. The process begins by using standard photoresist and optical lithography to define the large-area contact pads which consist of a 6 nm-thick $\mathrm{Cr}$ adhesion layer and a $80 \mathrm{~nm}$-thick Au film. The substrates are then coated with a PMMA thin film, which is then patterned by electron beam lithography into a Ni lift-off mold that defines the submicron mechanical components of the $\mathrm{SiC}$ devices. The patterned Ni mask $(\sim 36 \mathrm{~nm}$ thick) is transferred to the $3 \mathrm{C}-\mathrm{SiC}$ layer by electron cyclotron resonance (ECR) etching using a $\mathrm{NF}_{3} / \mathrm{O}_{2} / \mathrm{Ar}$ anisotropic etch. The newly patterned $3 \mathrm{C}-\mathrm{SiC}$ beams are then released by simply etching the underlying $\mathrm{Si}$ substrate using an isotropic $\mathrm{NF}_{3} / \mathrm{Ar}$ ECR etch. The Ni mask remains on the $\mathrm{SiC}$ beams to be used as a conducting

Travel support for X. Huang has been generously provided by the Transducers Research Foundation and the DARPA MEMS and DARPA BioFlips Programs.

\author{
M.L. Roukes \\ Condensed Matter Physics, California Inst. of Tech. \\ Pasadena, California 91125
}

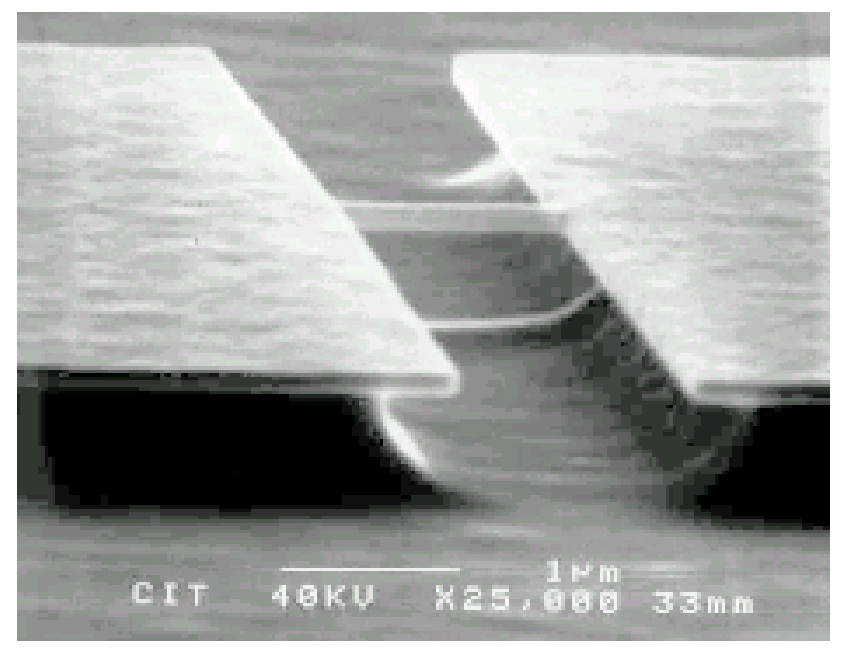

Figure 1. SEM showing one of the two nominally identical doubly-clamped SiC beams in a device structure. The SiC beam is capped with nickel metallization.

layer for device testing. The device structures consist of two identical doubly-clamped beams, as shown in Fig. 1. The beams are roughly $1.25 \mu \mathrm{m}$ long, $180 \mathrm{~nm}$ wide and $75 \mathrm{~nm}$ thick.

The test setup is shown in Fig. 2. The size of the beams prohibits use of electrostatic transduction, so magnetomotive transduction is instead employed. Each doubly-clamped beam pair is positioned perpendicular to a strong magnetic field (4 to 8T) in vacuum at cryogenic temperatures. An rf current is passed through the $\mathrm{Ni}$ conductor. For $\mathrm{rf}$ frequencies away from the mechanical resonances, the induced mechanical motion is minimal and the output at terminal III is ground. When the driving frequency matches the resonant frequency of one of the beams, motion will occur in that beam which induces an emf voltage at terminal III. After proper amplification, the voltage is measured by a network analyzer.

In the language of network analysis, we evaluate the mechanical performance of the device by measuring the frequency dependence of the forward transmission coefficient $S_{21}$ of the network. Information about the mechanical motion is revealed as resonance peaks in the spectra. The in-plane resonances are seen at $615 \mathrm{MHz}$ and $632 \mathrm{MHz}$, respectively, as shown in Figs. 3, where the frequency dependence of the forward transmission coefficient is plotted. The two resonances have a phase difference of about $180^{\circ}$, as shown in Fig. 4. These data contain information about both the mechanical resonator and the electrical connections. To extract information about the mechanical resonant structure, we subtract the background, which is also a complex-valued function of frequency, by fitting data points taken away from the resonance peaks. After subtracting the background, the amplitude of the resulting function can be plotted to clearly resolve the resonance peaks at $615 \mathrm{MHz}$ and $632 \mathrm{MHz}$, as shown in Fig. 5. 


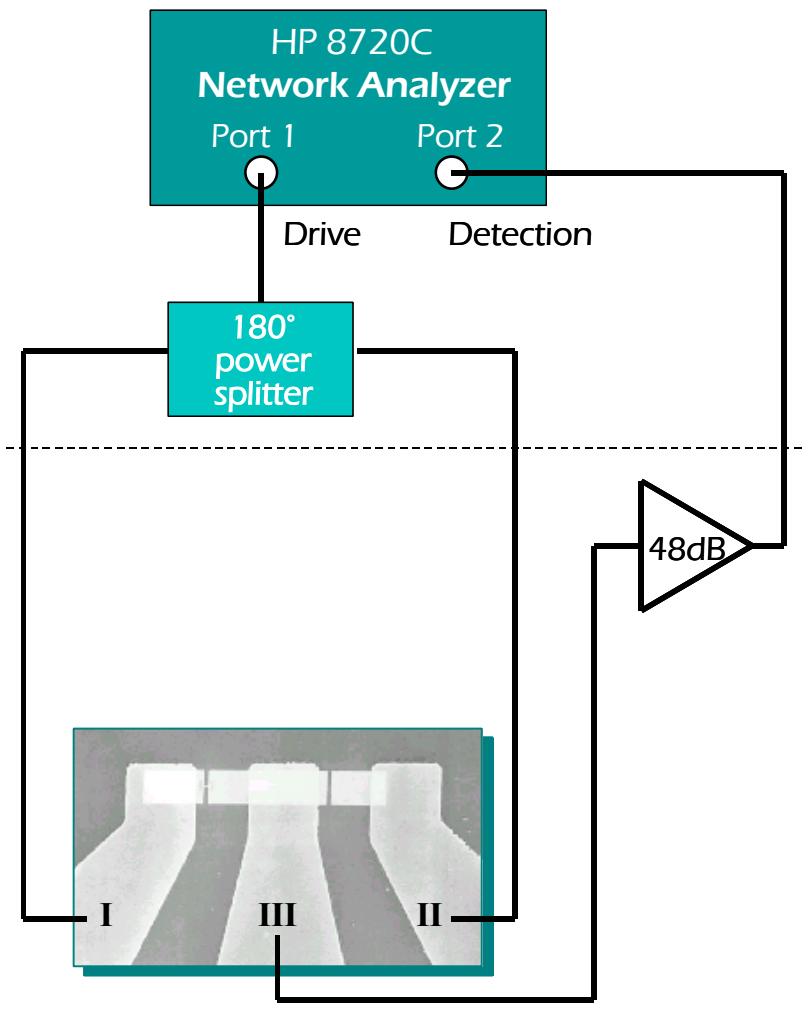

Figure 2. Schematic of the measurement setup. At the resonant frequency of one of the beams an emf is induced which induces a voltage. This emf voltage affects the power transmitted out from terminal III towards the detector. The power is amplified and detected at Port 2 of the network analyzer. The connections linked to pads I and II are identical from the device to the $180^{\circ}$ power splitter, which divides the driving power from Port 1 of the network analyzer into two equal partitions with a phase difference of $180^{\circ}$.

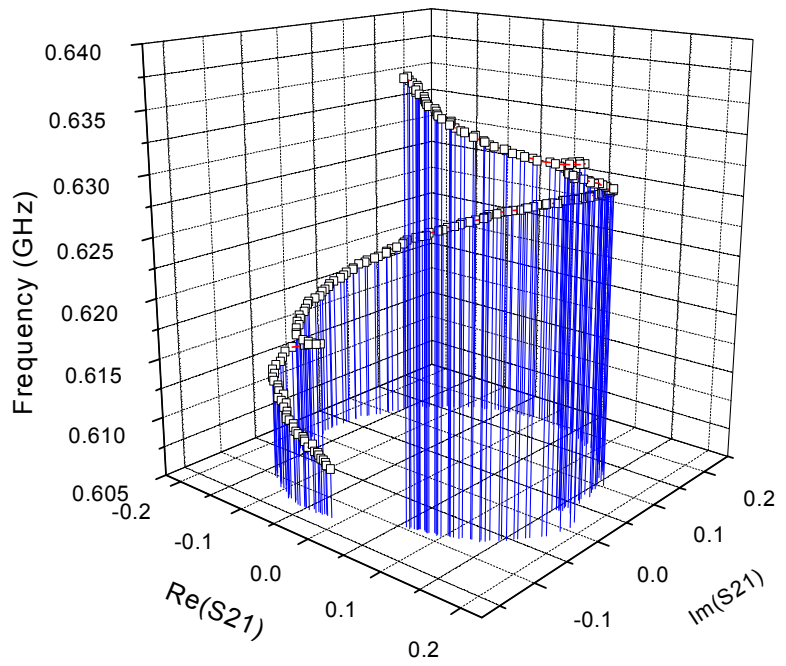

Figure 3. The frequency dependence of the forward transmission coefficient $S_{21}$ of the network. The magnetic field for the test is $8 \mathrm{~T}$, the driving power is $-60 \mathrm{dBm}$ and the resolution band width is $10 \mathrm{~Hz}$.

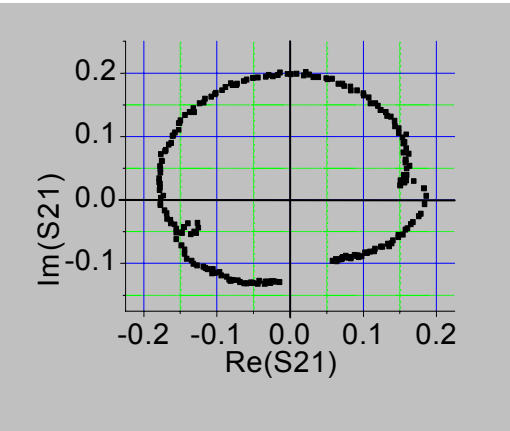

Figure 4. The projection of the complex function in Fig. 3 onto the $S_{21}$ plane, showing the $180 \mathrm{deg}$ phase difference in the two resonances.

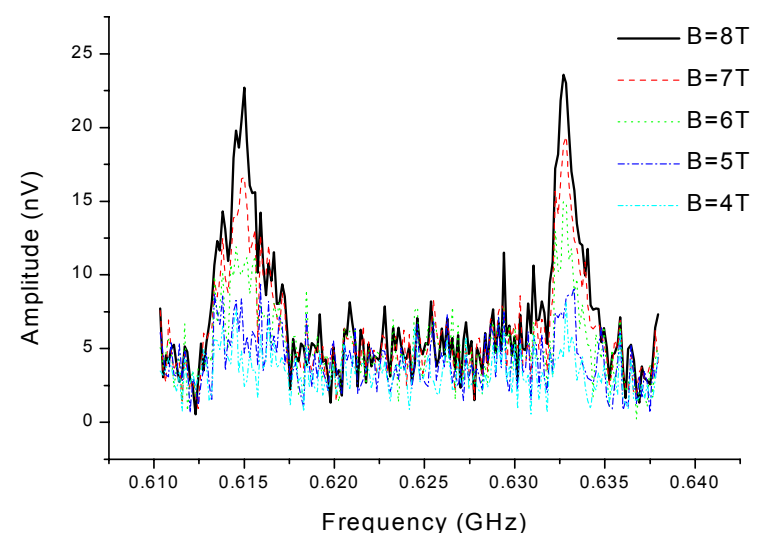

Figure 5. Signal amplitude referred back to the input of the pre-amplifier. This is obtained by taking modulus after subtracting the background function from the raw data.

\section{CONCLUSIONS}

We have demonstrated the measurement of $\mathrm{SiC}$ nanomechanical resonators with fundamental resonance frequencies in the UHF range, with clear promise of accessing the microwave L-band frequencies by the same technique. Exciting opportunities in physics and technology are anticipated from more detailed studies of such frequency bands of mechanical motion, which were inaccessible until now.

Acknowledgements: This work was supported by DARPA MTO/MEMS under grants DABT63-98-1-0012 (Caltech) and DABT63-98-1-0010 (CWRU).

\section{REFERENCES}

[1] M. Mehregany, C. Zorman, N. Rajan, and C. Wu, Proc. IEEE, 86, 1594, (1998).

[2] J. Wang, J. Butler, D Hsu, and C. Nguyen, Tech. Dig. $15^{\text {th }}$ Int. Conf. on MEMS, 657, (2002).

[3] M. Roukes, Technical Digest - 2000 Solid State Sensor and Actuator Workshop, Hilton Head SC, June 4-8, 2000, p. 367.

[4] Y. Yang, K. Ekinci, X. Huang, L. Schiavone, M. Roukes, C. Zorman, and M. Mehregany, Appl. Phys. Lett., 78, 162, (2001).

[5] C.A Zorman, A.J. Fleischman, A.S. Dewa, M. Mehregany, C. Jacob, and P. Pirouz, J. Appl. Phys, 78, 5136, (1995). 